\title{
Inclination Effects on Voltage-controlled Tuning of MEMS Disk Resonator Array Composite Fabricated by Deep Reactive-ion Etching
}

\author{
Haixia Yan, ${ }^{1}$ Jinyan Bao, ${ }^{2}$ Quan Yu, ${ }^{2}$ and Linxi Dong ${ }^{2,3,4^{*}}$ \\ ${ }^{1}$ Hangzhou Dianzi University Information Engineering School, Hangzhou 310018, China \\ ${ }^{2}$ The Key Laboratory of RF Circuits and System of Ministry of Education, College of Electronic and Information, \\ Hangzhou Dianzi University, Hangzhou 310018, China \\ ${ }^{3}$ State Key Laboratory of Functional Materials for Informatics, Chinese Academy of Sciences, \\ Shanghai 200050, China \\ ${ }^{4}$ State Key Laboratory for Manufacturing Systems Engineering, Xi'an Jiaotong University, Xi'an 710049, China
}

(Received May 2, 2018; accepted September 4, 2018)

Keywords: DRIE process, MEMS disk resonator array, electrostatic tuning, electromechanical coupling, motional resistance

Capacitive micro-electromechanical system (MEMS) disk resonators fabricated by deep reactive-ion etching (DRIE) have large sensitive capacitances and low motional resistances. However, for the MEMS disk structure with a high aspect ratio, the cross section takes on a trapezoidal profile, which will affect the performance of the resonator. In this study, we firstly analyzed the electrostatic tuning mechanism of electrical stiffness produced by the electrostatic force, and the dependence of resonance frequency variation on the inclination angle and dc bias voltage is obtained and the electromechanical coupling strength was changed owing to the inclination angle. Secondly, after analyzing the feasibility of overcoming the inclination effect by introducing a tuning disk array, the optimal tuning voltage of the inclined disk resonator array and the scale of the array with a small motional resistance can be obtained. The results show that, for an array with the same inclination angle of $0.1^{\circ}$ and biased at $10 \mathrm{~V}$, when the tuning voltage is $20 \mathrm{~V}$, the relative error of the resonance frequency can be reduced to $12.4 \mathrm{ppm}$. In addition, the optimal tuning voltage increases as the inclination angle increases, and when the inclination angle is $0.3^{\circ}$, the optimal tuning voltages are 28.68 and $57.35 \mathrm{~V}$ for the dc bias voltages of 10 and $30 \mathrm{~V}$, respectively. If the motional resistance needs be reduced to $50 \Omega$, the integrated number of disk resonators will increase to 1048, and the optimal tuning voltage can reach $37.5 \mathrm{~V}$. These results can provide some theoretical basis for the large-scale integration of the disk resonator array in the future.

\section{Introduction}

Oscillators based on micro-electromechanical system (MEMS) resonators have recently become viable alternatives to traditional quartz versions owing to their small size, high quality

*Corresponding author: e-mail: donglinxi@hdu.edu.cn https://doi.org/10.18494/SAM.2018.1972 
factor and single-chip integration with the CMOS process. ${ }^{(1,2)}$ Compared with other resonator types, vibrating MEMS capacitive disk resonators have more applications because of recent advances that yield on-chip resonators with Qs over 40000 at $3 \mathrm{GHz}^{(3,4)}$ and excellent thermal ${ }^{(5)}$ and aging stabilities. ${ }^{(6)}$

Unfortunately, although the Qs of the described resonators are exceptional, they are not easy to access, because impedances mismatch with systems using them..$^{(7,8)}$ One possible solution to this impedance problem is to use piezoelectric materials, such as AIN, whose impedance is below $80 \Omega$, and the Qs are below $2900 .{ }^{(9)}$ For a single MEMS resonator, increasing the sensing capacitance is also one of the methods to reduce the motional impedance, for example, increasing the thickness of a disk fabricated by deep reactive-ion eching (DRIE), ${ }^{(10,11)}$ or decreasing the electrode-to-resonator gap. ${ }^{(12)}$ However, the motional impedance increases linearly with increasing frequency, ${ }^{(13)}$ and when the frequency is in the ultrahigh frequency (UHF) range, the method of only increasing the thickness or reducing the gap is generally insufficient if a $50 \Omega$ matching impedance is desired.

To circumvent this, the design method of using coupled-array composite resonators that mechanically link individual disk resonators via half-wavelength coupling beams can be used. $^{(14)}$ Here, all resonators vibrate at precisely the same frequency, allowing their outputs to be combined to boost input and output currents, thereby decreasing the motional resistance and improving linearity by reducing the amplitude. ${ }^{(15)}$ Reference 16 shows that larger mechanically coupled arrays can offer better stability against electrical-stiffness-based frequency instability. For example, $\operatorname{Nguyen}^{(8)}$ showed that, at $1 \mathrm{GHz}, 50 \Omega$ can be achieved using a mechanically coupled array of 243-mm-thick, 2.64-mm-radius, and 50,000-Q poly-silicon disks, each with a dc bias voltage of $10 \mathrm{~V}$ and an electrode-to-resonator gap spacing of $30 \mathrm{~nm}$. Ideally, arraying can improve the linearity and motional resistance. However, owing to fabrication nonidealities, the random variation in the resonator and coupling beam dimensions may cause the array composites to be short of the expected impedance and linearity improvements. Akgul et al. ${ }^{(17)}$ demonstrated a voltage-controlled electrical stiffness tuning method to correct phase and amplitude mismatches between the constituent resonators.

However, previous studies were based on the ideal capacitor plate, without considering the effects of fabrication errors on the plate of the MEMS disk resonator arrays. In a DRIE process, the radius of the disk increases linearly with depth and the cross section takes on a trapezoidal profile, ${ }^{(18,19)}$ which leads to a frequency offset. ${ }^{(20)}$ Electrical stiffness tuning technology is a method for solving the problem. In this study, we firstly analyzed the stiffness tuning mechanism and showed the dependence of resonator frequency on inclination angle and dc bias voltage by curves. Secondly, we introduced a tuning disk array to change the original resonator frequency and obtained the optimal tuning voltage and size of the tuning disk array.

\section{Electrostatic Tuning Mechanism of Electrical Stiffness}

Figure 1 shows the schematic of the radial-contour mode disk resonator without considering the inclination. The resonator consists of a disk above the substrate with a single anchor at its center. Input and output electrodes surround the perimeter of the disk, separated from it by a 


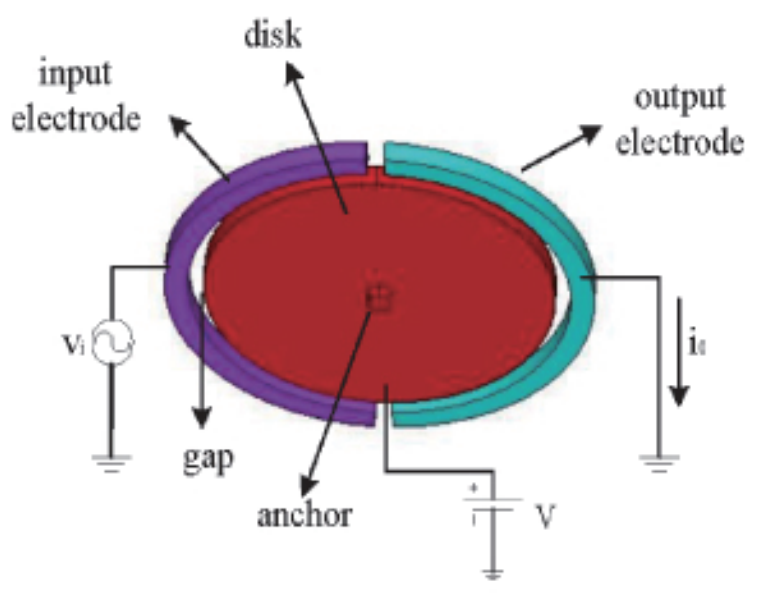

Fig. 1. (Color online) Diagram of disk resonator.

narrow air gap, which defines the capacitive MEMS resonator of this device. ${ }^{(21)}$ To stimulate the device shown in Fig. 1, a dc bias voltage $(V)$ is applied to the disk and an AC signal to its input electrode. Together, these voltages generate time-varying electrostatic force acting radially on the disk. When the frequency matches the radial-contour mode resonance frequency of the device, the electrostatic force drives the disk to expand and contract radially around its perimeter. The disk resonator can be represented by a mechanical model, which is composed of a damper element, a rigid-body mass, and a spring, as shown in Fig. 2, where $k_{r}$ is the purely mechanical stiffness, $m_{r e}$ is the lumped equivalent mass, and $c_{r e}$ is the damping coefficient.

Under the action of the radial electrostatic force, the dynamic equation of the disk resonator can be expressed as ${ }^{(22)}$

$$
m_{r e} \frac{\partial^{2} r}{\partial t^{2}}+c_{r e} \frac{\partial r}{\partial t}+k_{r} r=F_{\text {elec }}
$$

where $r$ is the radial deformation of the disk. Regardless of the small signal AC voltage $\left(v_{i}<<V\right)$, the electrostatic force $F_{\text {elec }}$ is given by

$$
F_{\text {elec }}=\frac{\varepsilon_{0} A V^{2}}{2 d^{2}}
$$

where $\varepsilon_{0}$ is the permittivity of vacuum, $A$ and $d$ are the overlapping area and initial capacitive distance between the disk and the electrodes, respectively, and $V$ is the dc bias voltage. Assuming that $r<<d$, Eq. (1) can be expanded as

$$
m_{r e} \frac{\partial^{2} r}{\partial t^{2}}+c_{r e} \frac{\partial r}{\partial t}+k_{r} r=\frac{\varepsilon_{0} A V^{2}}{2(d-r)^{2}} \approx \frac{\varepsilon_{0} A V^{2}}{2 d^{2}}\left(1+\frac{2 r}{d}\right) .
$$




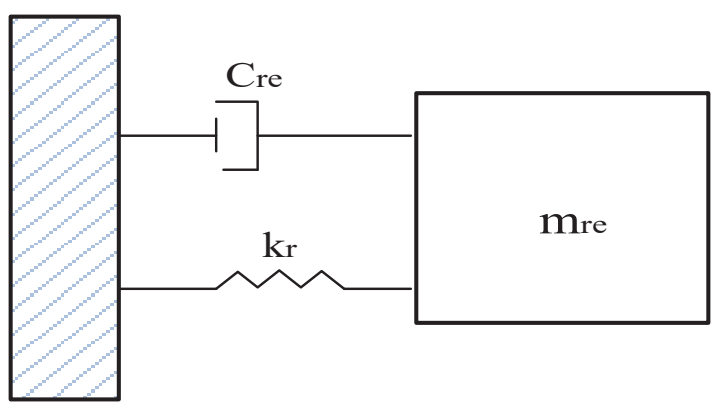

Fig. 2. Equivalent mechanical model of MEMS disk resonator.

Equation (3) can be converted into

$$
m_{r e} \frac{\partial^{2} r}{\partial t^{2}}+c_{r e} \frac{\partial r}{\partial t}+\left(k_{r}-\frac{\varepsilon_{0} A V^{2}}{2 d^{3}}\right) r=\frac{\varepsilon_{0} A V^{2}}{2 d^{2}}=F_{\text {elec }},
$$

and the electrical stiffness can be expressed as

$$
k_{e}=\frac{\varepsilon_{0} A V^{2}}{d^{3}} .
$$

Hence, the resonance frequency changes to

$$
\sqrt{\frac{k_{r e}}{m_{r e}}}=\frac{1}{2 \pi} \sqrt{\frac{k_{r}-k_{e}}{m_{r e}}} .
$$

As shown in Eq. (6), the electrical stiffness reduces the frequency of resonator. Therefore, the resonance frequency can be tuned by changing the electrical stiffness, and the efficacy of the tuning method can be evaluated by selecting suitable $V$ and $d$ values.

\section{Inclination Effects on Resonance Frequency of Single Disk Resonator}

Actually, the disk structure with a high aspect ratio fabricated by DRIE can reduce the motional resistance. However, owing to the DRIE error, the disk and electrodes are usually not ideally parallel, and the cross section takes on a trapezoidal profile, as shown in Fig. 3. Assuming that the bottom surface of the disk shows the design value and the electrode-todisk gap is $d$, the disk and electrodes have the same thickness $h$ with the inclination angle $\theta$. If voltage is not applied to the resonator, the purely mechanical stiffness $k_{r}$ is given by 


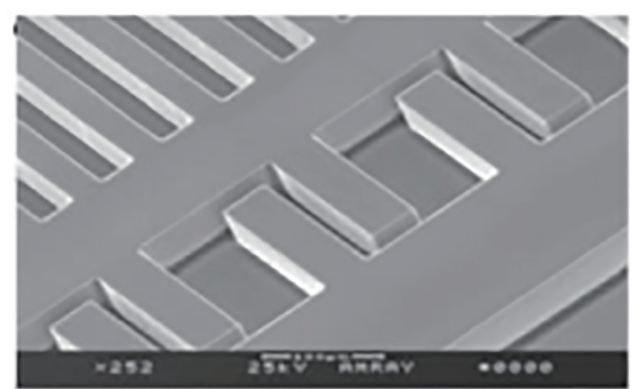

(a)

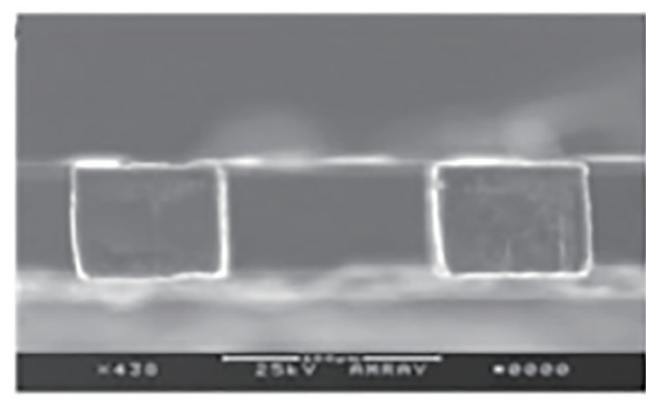

(b)

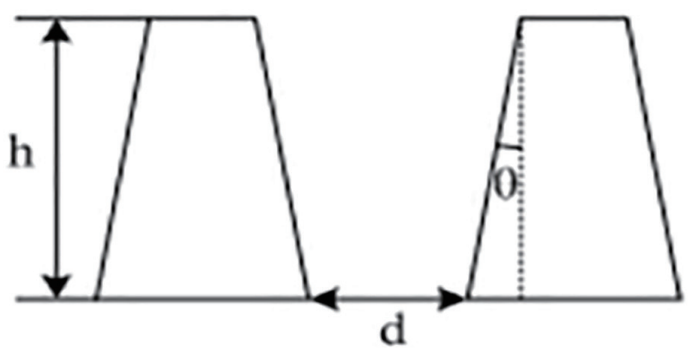

(c)

Fig. 3. (a) Comb sensing capacitors fabricated by DRIE. (b) Nonideality profiles of combs with micrometer-order distance and (c) cross-sectional diagram of nonparallel comb.

$$
k_{r}=\left(2 \pi f_{0}\right)^{2} m_{r e}
$$

where $f_{0}$ is the inherent frequency and its value can be obtained by theoretical calculation or ANSYS simulation. $m_{r e}$ is the equivalent mass of the disk. When considering the inclination, it can be expressed as ${ }^{(23)}$

$$
m_{r e}=2 \pi \rho \int_{0}^{h} \frac{d x}{J_{1}^{2}\left[h_{1}(R-x \tan \theta)\right]} \int_{0}^{R-x \tan \theta} r J_{1}^{2}\left(h_{1} r\right) d r,
$$

where $\rho$ is the disk density, $J_{1}(x)$ is the Bessel function of one order, and $h_{1}$ is a constant related to the material

$$
h_{1}=2 \pi f_{0} \sqrt{\frac{\rho}{\frac{E}{1+\sigma}+\frac{E \sigma}{1-\sigma^{2}}}},
$$

where $E$ is the Young's modulus and $\sigma$ is the Poisson's ratio. When a dc bias voltage $V$ is applied to the resonator, the electrostatic force between the disk and the electrodes enables the electrical stiffness to be changed as ${ }^{(24)}$ 


$$
k_{e 1}=\frac{\pi \varepsilon_{0} V^{2} \cos \theta}{2 \theta} \cdot\left[\frac{(4 R+2 d) h \tan \theta}{d^{3}+2 h d^{2} \tan \theta}-\frac{(4 R+2 d) h^{2}(\tan \theta)^{2}}{\left(d^{2}+2 h d^{2} \tan \theta\right)^{2}}-\frac{2 h \tan \theta}{d^{2}+2 h d \tan \theta}\right] .
$$

The practical resonance frequency is given by

$$
f(\theta, V)=f_{0} \sqrt{1-\frac{k_{e 1}}{k_{r}}} \approx f_{0}\left(1-\frac{1}{2} \frac{k_{e 1}}{k_{r}}\right) .
$$

The relative amount of resonance frequency change can be expressed as

$$
\frac{\Delta f}{f}=\frac{\sqrt{k_{r}-k_{e 1}}-\sqrt{k_{r}-k_{e}}}{\sqrt{k_{r}-k_{e}}} .
$$

For a certain disk resonator, the parameters, such as $R$ and $h$, have certain values. Owing to the inclination caused by DRIE and stiffness hardening effects, with the increasing effect of inclination, the purely mechanical stiffness $k_{r}$ will increase, the electrical stiffness $k_{e 1}$ will decrease, and the practical resonance frequency $f(\theta, V)$ will clearly increase. Fortunately, the resonance frequency can be tuned by the electrostatic spring effect produced by the dc bias voltage $V$. In the following sections, we will analyze the resonance frequency change of a certain single disk resonator. Table 1 lists the specific sizes of the disk resonator.

The ANSYS-simulated resonance vibration mode shape for a radial-contour mode disk resonator with an inclination angle $0.1^{\circ}$ is shown in Fig. 4. Through the simulation, the natural resonator frequency $f_{0}$ for different inclinations is shown in Fig. 5. With a change in inclination angle from 0.1 to $0.3^{\circ}$, the resonance frequency increases by $0.03 \%$. Furthermore, according to Eqs. (7) and (9), some parameters can be obtained. For example, for a $0.1^{\circ}$-inclined resonator, the effective mass is $2.294 \times 10^{-11} \mathrm{~kg}$ and the purely mechanical stiffness is $7.028 \times 10^{6} \mathrm{~N} / \mathrm{m}$. In addition, for different inclination angles, the resonance frequency can be tuned by a different dc bias voltage, as shown in Fig. 6, showing that, as the dc bias voltage changes from 10 to 16 $\mathrm{V}$, the frequency shift of the $0.1^{\circ}$-inclined resonator increases from 12.4 to $31.8 \mathrm{ppm}$, and that of the $0.3^{\circ}$-inclined resonator increases from 28.5 to $72.9 \mathrm{ppm}$.

Table 1

Geometric sizes of the disk resonator.

\begin{tabular}{lc}
\hline Geometric size & Design value $(\mu \mathrm{m})$ \\
\hline Radius of disk & 32 \\
Disk-electrode gap & 80 \\
Thickness of disk & 3 \\
Radius of anchor & 1.5 \\
Thickness of anchor & 1 \\
\hline
\end{tabular}




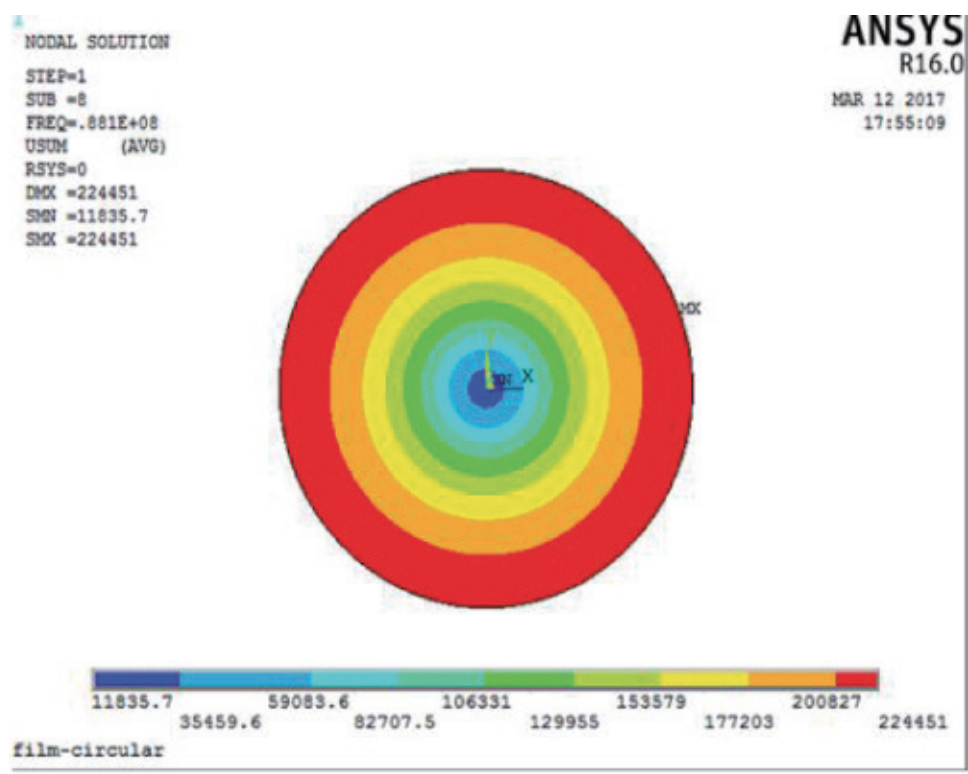

Fig. 4. (Color online) ANSYS-simulated resonance vibration mode shape for a radial-contour mode disk resonator.

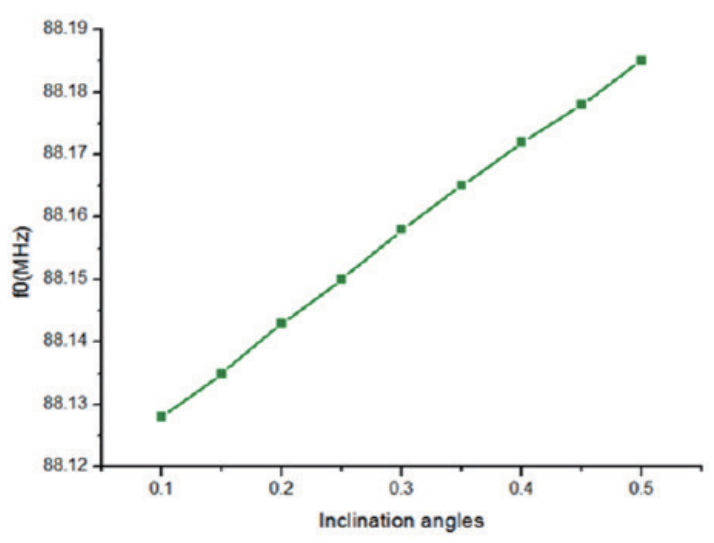

Fig. 5. (Color online) Natural resonator frequency versus inclination angle.

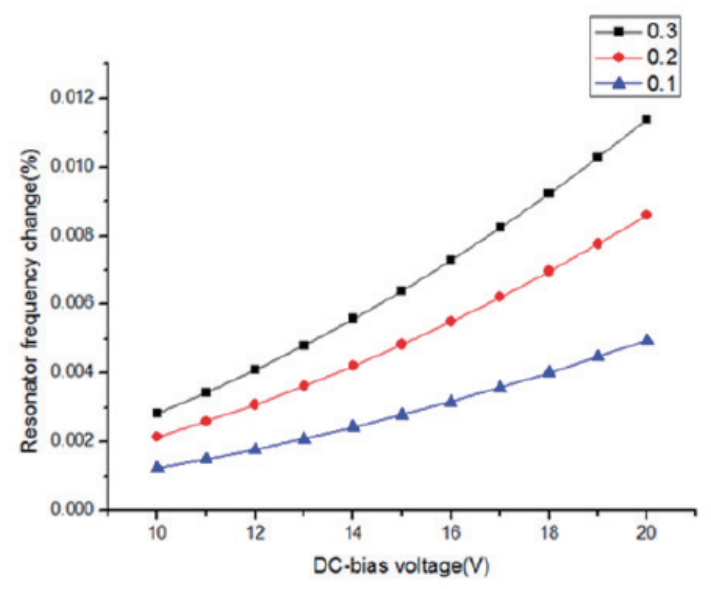

Fig. 6. (Color online) Resonance frequency versus $\mathrm{dc}$ bias voltage with different inclination angles.

In addition, Fig. 7 shows the plot of fractional frequency change versus inclination angle for a disk resonator biased at a dc bias voltage of $10 \mathrm{~V}$. As shown by the curve, the resonance frequency increases from 12.4 to $28.5 \mathrm{ppm}$ as the inclination angle changes from 0.1 to $0.3^{\circ}$. This deviation is so large that the effect of the inclination cannot be ignored. 


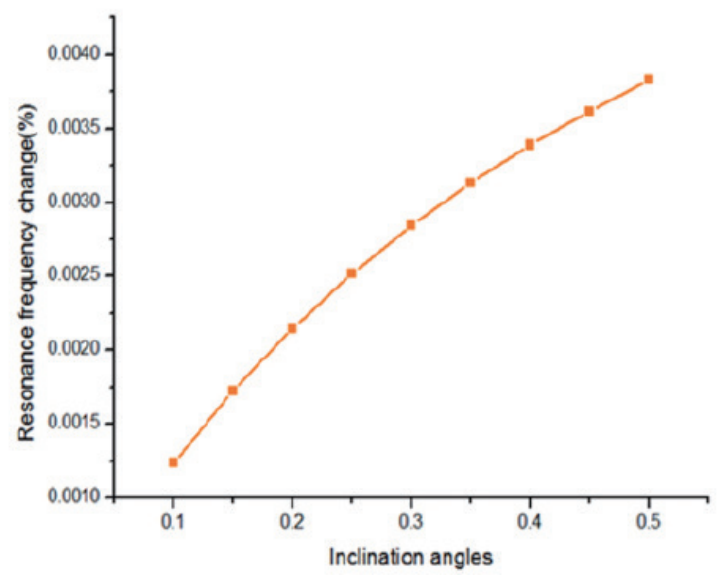

Fig. 7. (Color online) Resonance frequency change versus inclination angle.

\section{Inclination Effects on Electromechanical Coupling Strength}

In the process, the gap will be subject to linearity constraints. Maybe, the gap is too large to affect the electromechanical conversion and energy transfer, or if the gap is too small and the bias voltage is large, it will cause the pull-in effect, and the third-order intermodulation distortion of the resonator (IM3) will increase. ${ }^{(25)}$ When the disk resonators are connected by a quarter-wavelength beam, the MEMS resonators can form a composite disk array filter. For today's commonly used 3\% band-pass filters, more than $7 \%$ of the electromechanical coupling strength is often needed. If only a few filters are needed, it would be good to avoid the addition of the inductor, and the filter attains sufficient electromechanical coupling strength on its own at best. ${ }^{(26)}$ When the distance between the electrode and the mechanical oscillator is reduced to 39 $\mathrm{nm}$, the input/output electromechanical coupling effect can be enhanced 8.6 times according to Akgul et al. ${ }^{(27)}$ This part is mainly studied by considering the process error of the relationship between the electromechanical coupling strength and the gap. For convenience, the capacitor gap is calculated from the average. Therefore, the electromechanical coupling factor affected by the process after the electromechanical coupling strength can be expressed as

$$
\frac{C_{x}}{C_{0}}=\frac{V^{2}}{(d+h \tan (\theta))^{3}} \frac{n \varepsilon_{0} R \theta_{r}}{m \chi \pi^{3} k E},
$$

where $\theta_{r}$ is the arc of the electrode around the disk resonator. From Fig. 1, except for a slight loss of coupling beam connections, the electrodes are surrounded by the disk, $\theta_{r} \approx 2 \pi ; \chi$ represents the relationship between the dynamic mass and the static mass, assuming that the disk vibrates in the eighth mode, $\chi \approx 1 ; K$ has a relationship with the material's Poisson's ratio, for polysilicon, $K=0.654$. $n$ is the total number of disks and $m$ is the number of disks with driven input/output electrodes. If $m=n$, the array does not change the coupling strength. As can be seen from Eq. (13), once the filter structure design is determined, the electromechanical 
coupling strength can only be adjusted by changing the dc bias voltage and disk electrode gap. ${ }^{(27)}$ Table I shows the specific size of the disk resonator and the effects of tilt angle and dc bias voltage on the electromechanical coupling strength are shown in Fig. 8.

The diagrams present that $C_{x} / C_{0}$ decreases with increasing slope angle, that is, when the dc bias voltage $V$ is $15 \mathrm{~V}, C_{x} / C_{0}$ decreases to $6.38,3.72$, and $2.35 \%$ as the slope angle increases from 0.10 and 0.12 to $0.14^{\circ}$, respectively. Fortunately, the dc bias voltage can enhance the coupling strength to some degree as seen in Fig. 9. However, with the deviation of the angle, the subsidy becomes weaker. Moreover, the dc bias voltage cannot increase endlessly. Besides, when $V$ is $20 \mathrm{~V}, C_{x} / C_{0}$ decreases from 11.34 to $6.38 \%$ with the slope angle increase from 0.1 to $0.15^{\circ}$, which means that $C_{x} / C_{0}$ will not satisfy $3 \%$ band-select filters, and results in insufficient stopband rejection. It is obvious that the effect of the slope angle cannot be ignored.

\section{Electrostatic Tuning of the Disk Resonator Array}

The mechanical-coupled resonator can not only increase the output current, and raise the power handling, but also increase or decrease the number of disks to adjust the motion resistance. ${ }^{(7)}$ However, for most applications, e.g., filters, the resonance frequency change caused by the inclination effect can significantly reduce the frequency selectivity, and frequency tuning is necessary. Reference 17 provides an effective method of regulating the resonance frequency of the original array by introducing a tuning disk array, and the efficacy of the tuning method can be evaluated by selecting a suitable tuning voltage. Figure 10 presents the schematic of the resonator array with the tuning disk.

If the disks are coupled by half-wavelength beams, all resonators will vibrate at the same frequency. For the $(N+M)$ resonator array, where $M$ resonators are used for input/output and $N$ resonators for frequency tuning, the expression of the frequency of the array composite becomes

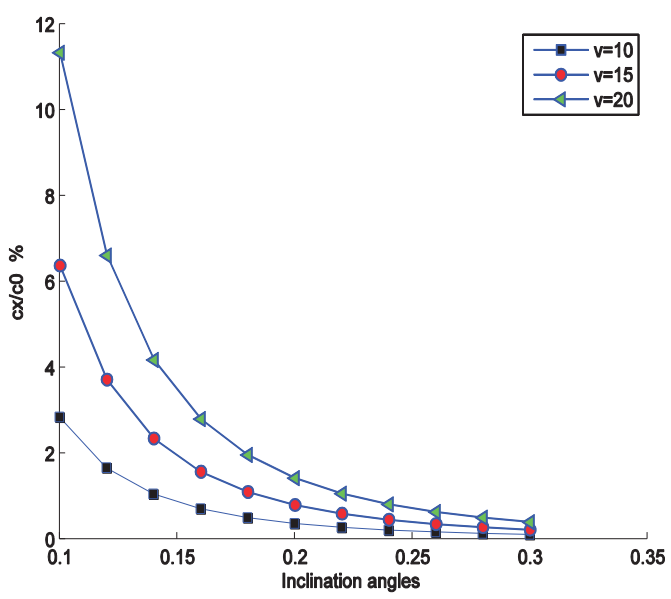

Fig. 8. (Color online) Slope angle change versus electromechanical coupling factor.

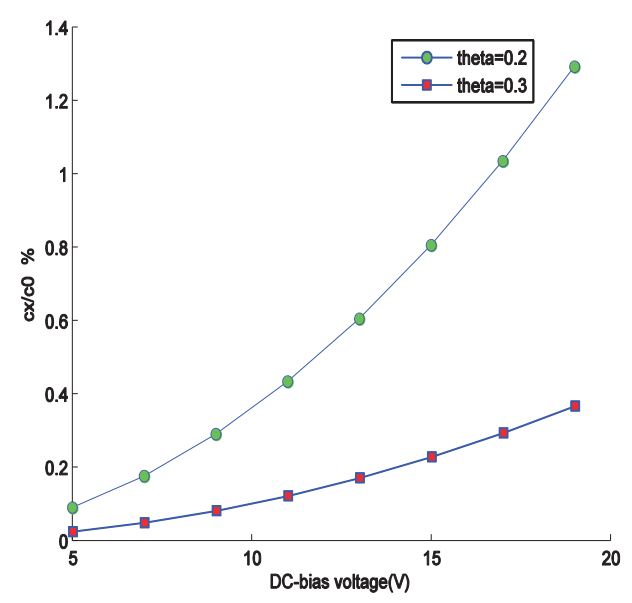

Fig. 9. (Color online) DC-bias voltage versus electromechanical coupling factor in a two-slope angle. 


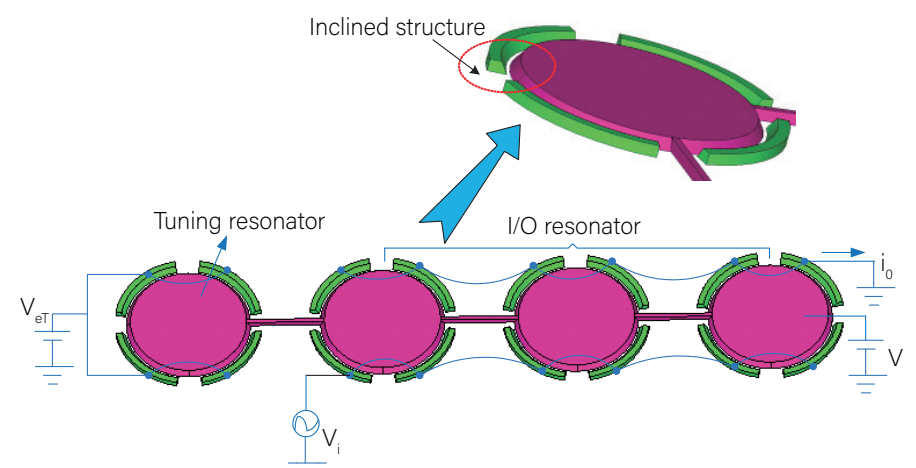

Fig. 10. (Color online) Schematic of the resonator array with the tuning disk.

$$
f_{\text {array }}=\frac{1}{2 \pi} \sqrt{\frac{(M+N) k_{r}-M k_{e 1}-N k_{e T}}{(M+N) m_{r e}}} .
$$

As described above, the measured resonance frequency of the array is higher than the theoretical value owing to the inclination effect, and the dc voltage difference in the tuning array is $\left(V_{e T}-V\right)$, as shown in Fig. 10. For effectively lowering the resonance frequency of the array by adjusting the tuning voltage, the electrical stiffness must satisfy $k_{e T}>k_{e l}$, according to Eq. (15), which can be expressed as

$$
\left|V_{e T}-V\right|>V \Rightarrow V_{e T}>2 V \text { or } V_{e T}<0
$$

In addition, Eq. (14) can be simplified as

$$
\begin{aligned}
& f_{\text {array }}=f_{0} \sqrt{1-\frac{M k_{e 1}+N k_{e T}}{(M+N) k_{r}}}, \\
& \approx f_{0}\left(1-\frac{1}{2} \cdot \frac{M k_{e 1}+N k_{e T}}{(M+N) k_{r}}\right) .
\end{aligned}
$$

With the same bias voltage and tuning disk array, the change in disk array frequency relative to the ideal resonator frequency is given by

$$
\frac{\Delta f}{f}=\frac{\sqrt{M+N} \cdot \sqrt{k_{r}-k_{e}}-\sqrt{(M+N) k_{r}-\left(M k_{e}+N k_{e 1}\right)}}{\sqrt{M+N} \cdot \sqrt{k_{r}-k_{e}}} .
$$

According to Eqs. (14), (15), and (18), the efficacy of the tuning method can be evaluated by selecting a suitable tuning voltage and specifying the number of tuning disks. 
Here, a resonator array composite with three disk resonators and one tuning resonator is taken as an example to analyze the inclination effects on the electrostatic tuning. For the array with the same inclination angle of $0.1^{\circ}$ and biased at $10 \mathrm{~V} \mathrm{dc}$, the resonance frequency error relative to the design value versus tuning voltage is shown in Fig. 11. The plot indicates that the relative error is reduced by increasing the tuning voltage. When the tuning voltage is $20 \mathrm{~V}$, the relative error of the resonance frequency of the array resonators is reduced to $12.4 \mathrm{ppm}$.

The tuning voltage that adjusts the resonance frequency of the fabricated resonators to the design value is defined as the optimal tuning voltage. The optimal tuning voltage can change with the inclination angle of the disk. Figure 12 presents the optimal tuning voltage versus inclination angle for the array with different bias voltages. As shown in Fig. 12, the optimal tuning voltage increases as the inclination angle increases, and when the inclination angle is $0.3^{\circ}$, for the dc bias voltages of 10 and $30 \mathrm{~V}$, the optimal tuning voltages are 28.68 and $57.35 \mathrm{~V}$ respectively.

For improving the output current of the disk array, increasing the scale of the I/O resonator array is necessary, which can change the optimal tuning voltage. For an array with the same inclination angle of $0.3^{\circ}$ and biased at $10 \mathrm{~V} \mathrm{dc}$, when the number of tuning disk resonators, $N$, is 1 , 2, or 3, the optimal tuning voltage versus the number of $\mathrm{I} / \mathrm{O}$ resonators $(M)$ is shown in Fig. 13. The plot indicates that the optimal tuning voltage increases as $M$ increases. For example, for an array composite with one tuning resonator and nine I/O resonators, the optimal tuning voltage is $27.34 \mathrm{~V}$. In addition, the optimal tuning voltage decreases as $N$ increases, for example, when $M=6$ and $N$ changes from 1 to 3 , the optimal tuning voltage decreases by $12.58 \%$.

\section{Inclination Effects on Motional Resistance of Scale Integration Disk Resonators}

Actually, for achieving a very low motional resistance while suppressing unwanted modes, the MEMS disk resonators can be designed by scale integration, such as in Fig. 14.

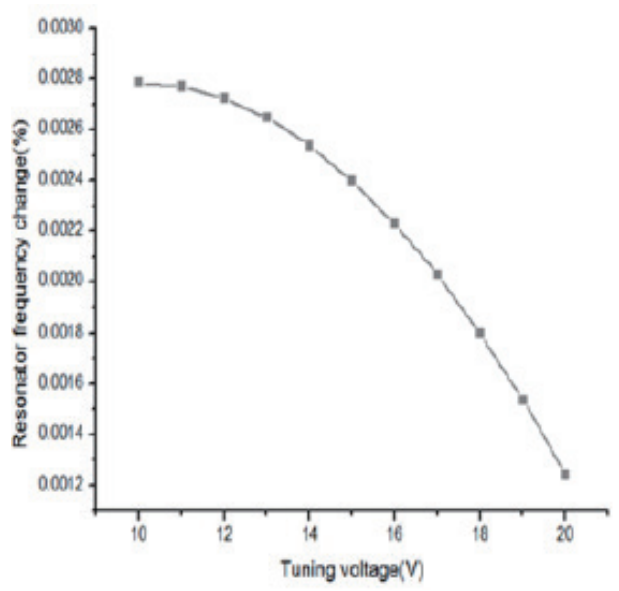

Fig. 11. (Color online) Resonance frequency error of array resonators relative to the design value versus tuning voltage.

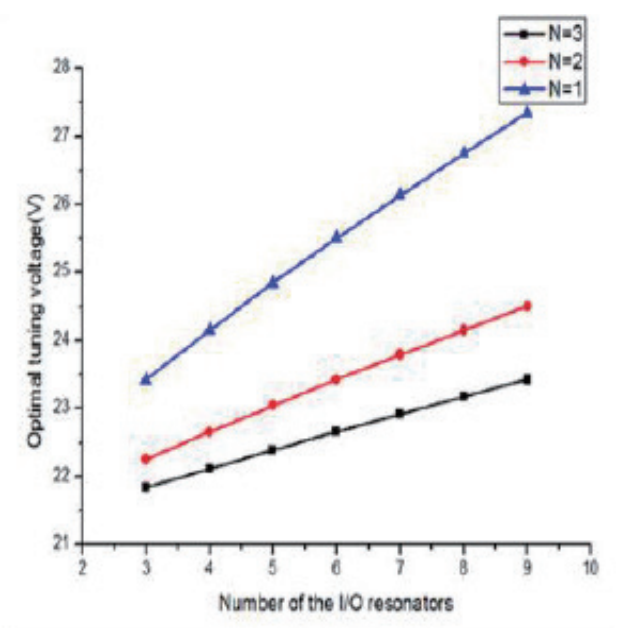

Fig. 12. (Color online) Optimal tuning voltage versus inclination angle. 


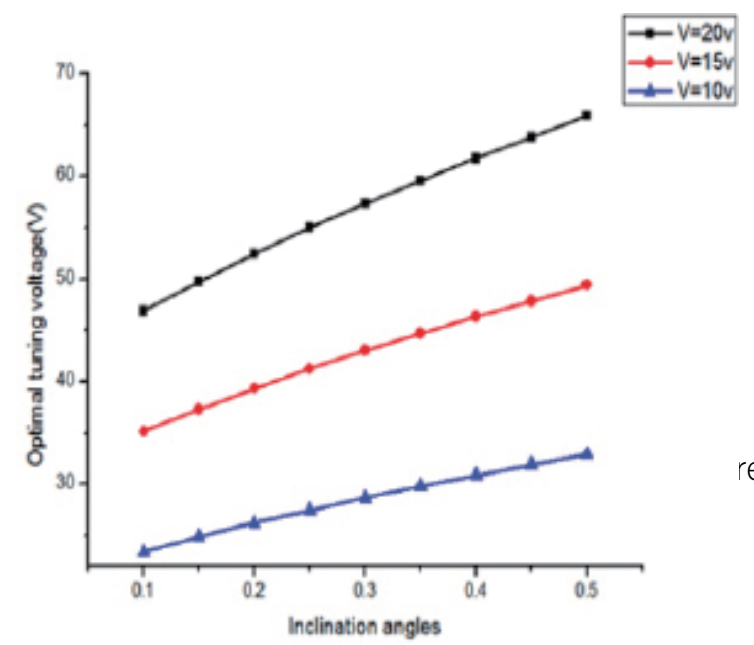

Fig. 13. (Color online) Optimal tuning voltage versus the number of $\mathrm{I} / \mathrm{O}$ resonators.

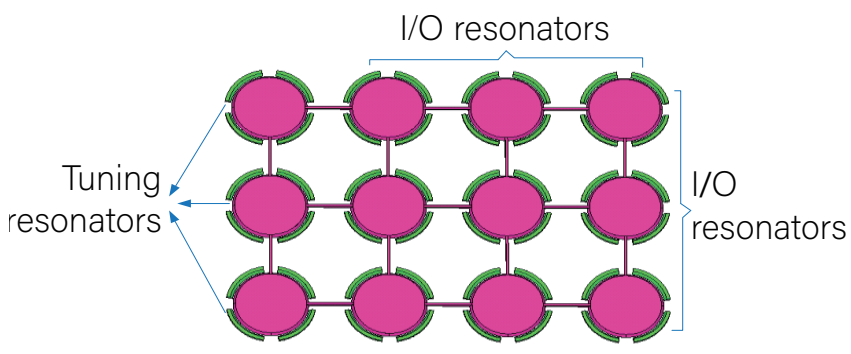

Fig. 14. (Color online) Schematic of a resonator array.

For matching the impedance of the front-end RF circuit, the motional resistance should be $50 \Omega$. For an array composite with $\mathrm{M} \mathrm{I} / \mathrm{O}$ resonators, the motional resistance can be expressed as

$$
R_{x}=\frac{1}{M} \cdot \frac{k_{r}(d+h \tan \theta)^{2}}{\omega_{0} Q C_{1}^{2} V^{2}}
$$

where $\omega_{0}$ is the radian resonance frequency, $Q$ is the quality factor, and $C_{1}$ is the disk electrodecapacitance, and it is given by

$$
C_{1}=\frac{\varepsilon_{0} \pi}{2 \theta}\left[\left(R+\frac{d}{2}\right) \ln \left(1+\frac{2 h \tan \theta}{d}\right)-h \tan \theta\right] .
$$

By using a disk array composite specified in Table 1 as an example for analyzing the feasibility of a large-scale integrated scheme of disks, the motional resistance of the disk resonator array can be calculated using Eq. (19). Because the quality factor of vibration micromechanical disk resonators is relative to the air damping, anchor loss, surface effect, and thermoelastic loss, its theoretical value is very difficult to obtain. Here, the quality factor is assumed as 10000 . When the inclination angle is $0.1^{\circ}$, from Eqs. (19) and (20), it is found that $C_{1}=0.0314 \mathrm{pF}$ and the motional resistance of each disk is $52400 \Omega$. In order to reduce the motional resistance to $50 \Omega$, the number of disk resonators should be 1048 with 32 disk resonators per row. When the array needs to be tuned and each row has only one tuning disk, the optimal tuning voltage reaches $37.5 \mathrm{~V}$. Therefore, for an array with a certain motional resistance of $50 \Omega$, the inclination angle of the disk will change the scale of the I/O resonator array and the optimal tuning voltage. 


\section{Conclusions}

The inclination effects on the electrostatic tuning of the capacitive MEMS disk resonator array have been studied in this work. How the electrical stiffness produced by the electrostatic force tunes the resonance frequency has been analyzed by simplifying the disk resonator as a mechanical model. For a single disk resonator, considering the inclination effects caused by DRIE, the changes in resonance frequency and electromechanical coupling strength have been given with several curves. After introducing a tuning disk array to the $\mathrm{I} / \mathrm{O}$ array, the optimal tuning voltage of the inclined disk resonator array and the integration scale of the array with an optimized motional resistance of 50 have been analyzed. The results show that, for an array with the same inclination angle of $0.1^{\circ}$ and biased at $10 \mathrm{~V} \mathrm{dc}$, when the tuning voltage is $16 \mathrm{~V}$, the relative error of the resonance frequency is reduced to $22 \mathrm{ppm}$. If the motional resistance decreases to 50 , the integrated number of disk resonators will increase to 1048, and the optimal tuning voltage can reach $37.5 \mathrm{~V}$. The inclination has a significant effect on array composite disk resonators, which is one of the factors to be considered when designing a MEMS resonator fabricated by DRIE. These results can provide some theoretical basis for the large-scale integration of the capacitive disk resonator array in the future.

\section{Acknowledgments}

The authors acknowledge the support of this research by the National Natural Science Foundation of China under Grant Nos. 61871167 and 61376117 and the Key Research and Development Plan Project of Zhejiang Province under Grant No. 2018 C01036.

\section{References}

1 T. L. Naing, T. O. Rocheleau, E. Alon, and C. T.-C. Nguyen: Proc. European Frequency and Time Forum \& International Frequency Control Symp. (EFTF/IFC) (IEEE 2013) 562-565. https://doi.org/10.1109/EFTFIFC.2013.6702305

2 W. C. Chen, W. Fang, and S. S. Li: J. Microelectromech. Syst. 21 (2012) 688. https://doi.org/10.1109/ JMEMS.2012.2189360

3 T. L. Naing, T. Beyazoglu, L. Wu, M. Akgul, Z. Ren, T. O. Rocheleau, and C. T.-C. Nguyen: Proc. IEEE Int. Frequency Control Symp. (IEEE 2012) 1-6. https://doi.org/10.1109/FCS.2012.6243723

4 J. Wang, J. E. Butler, T. Feygelson, and C. T.-C. Nguyen: Proc. 17th IEEE Int. Micro Electro Mechanical Systems Conference (IEEE 2004) 821-824. https://doi.org/10.1109/MEMS.2004.1290666

5 W.-T. Hsu and C. T.-C. Nguyen: Proc. Tech. Digest, 2002 IEEE Int. Micro Electro Mechanical Systems Conf. (IEEE 2002) 731-34. https://doi.org/10.1109/MEMSYS.2002.984374

6 B. Kim, R. N. Candler, M. Hopcroft, M. Agarwal, W. T. Park, and T. W. Kenny: Proc. Dig. Tech. Papers, Transducers (IEEE 2007) 1965-1968. https://doi.org/10.1109/SENSOR.2005.1497485

7 S. S. Li, Y. W. Lin, Z. Ren, and C. T.-C. Nguyen: Proc. Dig. Tech. Papers of the 14th Int. Conf. Solid-State Sensors \& Actuators (Transducers'07) (IEEE2007) 307-311. https://doi.org/10.1109/SENSOR.2007.4300130

8 C. T. C. Nguyen: IEEE Commun. Mag. 51 (2013) 110. https://doi.org/10.1109/MCOM.2013.6495769

9 G. Piazza, P. J. Stephanou, and A. P. Pisano: J. Microelectromech. Syst. 15 (2006) 1406. https://doi.org/10.1109/ JMEMS.2006.886012

10 G. Wu, D. Xu, B. Xiong, and Y. Wang: IEEE Sens. J. 12 (2012) 2414. https://doi.org/10.1109/JSEN.2012.2191772

11 G. Wu, D. Xu, B. Xiong, and Y. Wang: J. Micromech. Microeng. 22 (2012) 025020. https://doi. org/10.1088/0960-1317/22/2/025020 
12 M. Akgul, B. Kim, Z. Ren, and C. T.-C. Nguyen: Proc. Tech. Digest, 2008 Solid State Sensor, Actuator, and Microsystems Wksp (IEEE 2010) 467-470.

13 Y. Lin, W.-C. Li, B. Kim, Y.-W. Lin, Z. Ren, and C. T.-C. Nguyen: Joint Meeting of the European Frequency and Time Forum and the IEEE International Frequency Control Symp. (EFTF/IFCS 2009) (IEEE 2009) 58-63. https://doi.org/10.1109/FREQ.2009.5168142

14 M. U. Demirci and C. T.-C. Nguyen: Proc. Dig. Tech. Papers Int. Conf. Solid State Sensors and Actuators and Microsystems, TRANSDUCERS '05, (IEEE 2005) 2131-2134. https://doi.org/10.1109/SENSOR.2005.1497525

15 M. U. Demirci and C. T. C. Nguyen: J. Microelectromech. Syst. 15 (2006) 1419. https://doi.org/10.1109/ JMEMS.2006.883588

16 L. Wu, M. Akgul, W. Li, Y. Lin, Z. Ren, T. Rocheleau, and C. T.-C. Nguyen: Proc. European Frequency and Time Forum (IEEE 2013) 547-550. https://doi.org/10.1109/EFTF-IFC.2013.6702298

17 M. Akgul, Z. Ren, and C. T.-C. Nguyen: Porc. Frequency Control and the European Frequency and Time Forum (FCS) (IEEE 2011) 1-6. https://doi.org/10.1109/FCS.2011.5977901

18 F. E. H. Tay, X. Jun, Y. C. Liang, V. J. Logeeswaran, and Y. Yufeng: J. Micromech. Microeng. 9 (1999) 283. https://doi.org/10.1088/0960-1317/9/4/301

19 L. Dong, L. Che, L. Sun, and Y. Wang: Sens. Actuators, A 121 (2005) 395. https://doi.org/10.1016/ j.sna.2005.03.048

20 Z. Y. Zhong, W. M. Zhang, G. Meng, and J. Wu: J. Microelectromech. Syst. 22 (2013) 865. https://doi. org/10.1109/JMEMS.2013.2248127

21 M. Akgul, L. Wu, Z. Ren, and C. T. C. Nguyen: IEEE Trans. Ultrason. Ferroelectr. Freq. Control. 61 (2014) 849. PMID:24801124, https://doi.org/10.1109/TUFFC.2014.2976

22 S. Sung, W.-T. Sung, C. Kim, S. Yun, and Y. J. Lee: IEEE/ASME Trans. Mechatron. 14 (2009) 446. https://doi. org/10.1109/TMECH.2009.2023985

23 J. R. Clark, W. T. Hsu, M. A. Abdelmoneum, and C. T. C. Nguyen: J. Microelectromech. Syst. 14 (2005) 1298. https://doi.org/10.1109/JMEMS.2005.856675

24 L. Dong, Q. Yu, J. Bao, and J. Tao: J. Micromech. Microeng. 24 (2014) 105009. https://doi.org/10.1088/09601317/24/10/105009

25 R. Navid, J. R. Clark, M. Demirci, and C. T.-C. Nguyen: Proc. Tech. Digest, IEEE MEMS Conf. (IEEE 2001) 228-231. https://doi.org/10.1109/MEMSYS.2001.906520

26 M. Akgul and C. T.-C. Nguyen: Proc. Frequency Control Symposium (FCS) (IEEE 2014) 1-6. https://doi. org/10.1109/FCS.2014.6860009

27 M. Akgul, B. Kim, Z. Ren, and C. T.-C. Nguyen: Proc. Tech. Digest, 2010 Solid-State Sensor, Actuator, and Microsystems Workshop (IEEE 2010) 467-470. 